\title{
Energy spectra of Sub-Surface Velocity Fields Beneath Faraday Waves
}

\author{
Raffaele Colombi $^{1 *}$, Niclas Rohde, Michael Schlüter ${ }^{1}$, Alexandra von Kameke $^{2}$ \\ ${ }^{1}$ Hamburg University of Technology, Institute of Multiphase Flows, Hamburg, Germany \\ ${ }^{2}$ Hamburg University of Applied Sciences, Department of Engineering and Production Management, \\ Hamburg, Germany \\ *raffaele.colombi@tuhh.de
}

\begin{abstract}
Faraday waves form on the surface of a fluid which is subject to vertical forcing, and are researched in a large range of applications. Some examples are the formation of ordered wave patterns and the controlled walking or orbiting of droplets (Couder et al. (2005); Saylor and Kinard (2005)). Moreover, recent studies discovered the existence of a horizontal velocity field at the fluid surface, called Faraday flow, which was shown to exhibit an inverse energy cascade and thus properties of two-dimensional turbulence (von Kameke et al., 2011, 2013, Francois et al., 2013). Additionally, three-dimensionality effects have been part of recent investigations in quasi-2D flows (both electromagnetically-driven (Kelley and Ouellette, 2011; Martell et al., 2019) or produced by parametrically-excited waves (Francois et al., 2014; Xia and Francois, 2017)). Furthermore, the occurrence of an inverse cascade in thick layers is also subject of current studies on the coexistence of 2D and 3D turbulence (Biferale et al., 2012; Kokot et al., 2017; Biferale et al., 2017). By performing 2D PIV measurements at horizontal planes beneath the Faraday waves, we recently showed that pronounced three dimensional flows occur in the bulk, with much larger spatial and temporal scales than those on the surface (Colombi et al., 2021), when the system is not shallow in comparison to typical length scales of the surface flow (fluid thickness exceeding half the Faraday wavelength $\lambda_{F}$ ). This in turn reveals that an inverse energy cascade and aspects of a confined 2D turbulence can coexist with a three dimensional bulk flow. In this work, 2D PIV measurements of the velocity fields are carried out at a vertical cross-section $x z$-plane and at four distinct horizontal $x y$-planes at different depths in Faraday waves. The results reveal that small and fast vertical jets penetrate from the surface into the bulk with fast accelerating bursts and strong momentum transport in the $z$-direction. Furthermore, the fraction of flow kinetic energy in the vertical direction is found to peak inside a layer of approximately $10 \mathrm{~mm}$ (one Faraday wavelength) below the fluid surface.
\end{abstract}

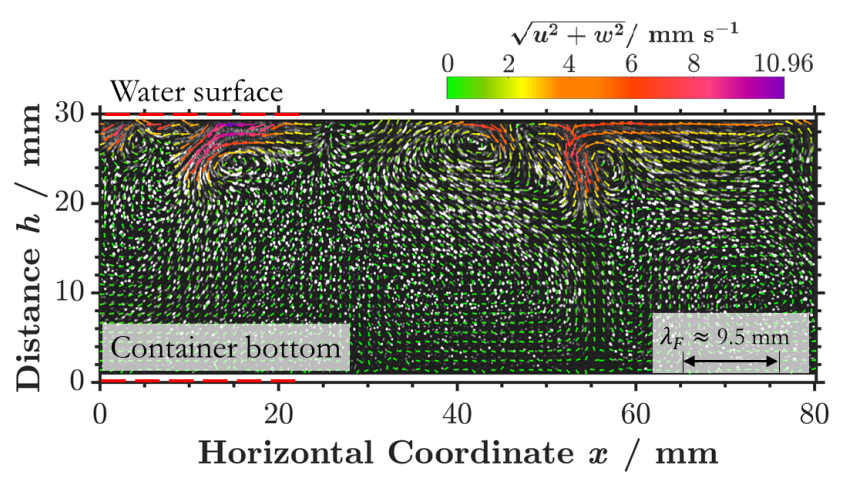

(a)

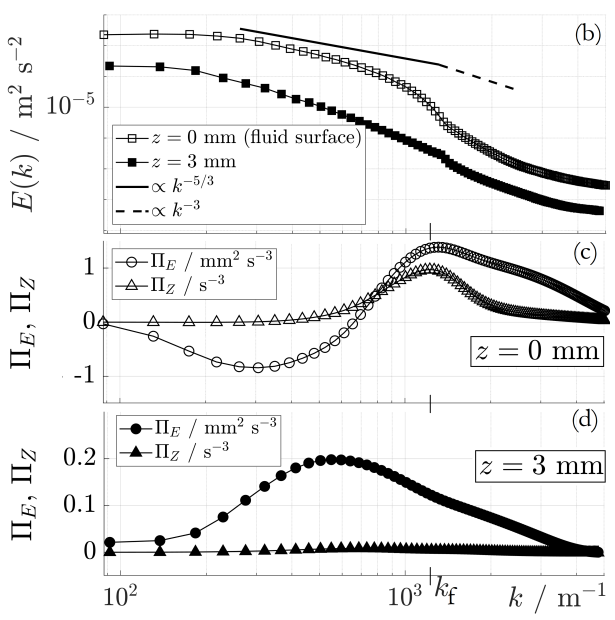

Figure 1: (a) Sub-surface velocity field in the $x z$-plane beneath Faraday waves (forcing frequency $f_{f}=50 \mathrm{~Hz}$, Faraday wavelength $\lambda_{F}=9.5 \mathrm{~mm}$ ). Neutrally buoyant, red fluorescent particles are used as fluid tracers. Fast vertical motion can be seen originating at the fluid surface. Background-image obtained by averaging 16 successive PIV frames (inverted). (Right) Wavenumber energy spectra of the horizontal velocity fields at the water surface $(z=0 \mathrm{~mm})$ and at depth $z=3 \mathrm{~mm}$. Inset (c) Net energy and enstrophy fluxes ( $\Pi_{E}$ and $\Pi_{Z}$ respectively) of the horizontal velocity fields at the water surface $(z=0 \mathrm{~mm})$ and at depth $z=3 \mathrm{~mm}$. The total depth of the water layer is $30 \mathrm{~mm}$. $k_{f}$ denotes the wavenumber at which the forcing occurs for $\lambda_{F} / 2$. 
Below this depth, the fast vertical motion dissipates in the bulk flow in a fashion similar to impinging jets on a flat surface, which might be the driving force of the bulk flow, see Fig. 1 a). This interpretation is supported by the analysis of energy spectra, as well as energy and enstrophy fluxes, see Fig. (1]b)-d), which show the existence of a direct energy cascade (as shown by the positive net energy flux $\Pi_{E}(k)$ and the zero net enstrophy flux $\Pi_{Z}(k)$, coexisting with the inverse energy cascade localized on the fluid surface. Finally, for the first time we perform time-resolved tomographic particle tracking velocimetry (4D PTV) in the bulk flow beneath the fluid surface, in order to further investigate the 3D transport of energy and its dissipation mechanisms from the horizontal surface flow to the bulk flow at lower depths. A schematic of the experimental setup is depicted and described in Fig. 2 a), whereas Fig. 2 b) depicts reconstructed 3D trajectories of particles in a slice at the container centre.
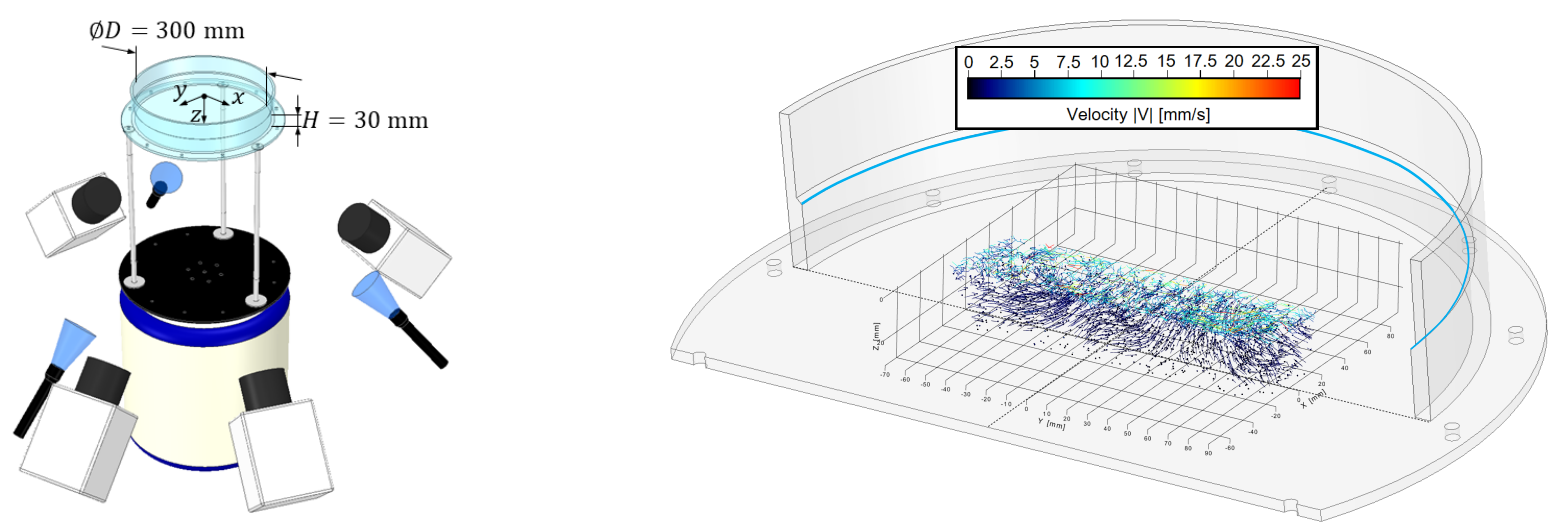

Figure 2: (Left) 4D PTV experimental setup. Four high-speed cameras are arranged in a tetrahedron configuration, and high-power LEDs are used to illuminate a volume of approx. $160 \times 160 \times 30 \mathrm{~mm}^{3}$ at the center of the vertically-agitated container $(\varnothing D=300 \mathrm{~mm})$, filled with $30 \mathrm{~mm}$ of DI water and a solution of neutrally-buoyant, red fluorescent particles $(150-180 \mu \mathrm{m})$. 4D PTV data is evaluated with the Flow Master tool (LaVision). (Right) Reconstructed 3D particle trajectories coloured with absolute velocity $|V|=\sqrt{u^{2}+v^{2}+w^{2}}$ in a volume slice of $40 \times 160 \times 30 \mathrm{~mm}^{3}$. The blue line represents the container filling height.

\section{Acknowledgements}

The authors gratefully acknowledge the financial support provided by the Deutsche Forschungsgemeinschaft (DFG) within the project 395843083 (KA 4854/1-1).

\section{References}

Biferale L, Buzzicotti M, and Linkmann M (2017) From two-dimensional to three-dimensional turbulence through two-dimensional three-component flows. Physics of Fluids 29:111101

Biferale L, Musacchio S, and Toschi F (2012) Inverse energy cascade in three-dimensional isotropic turbulence. Physical review letters 108:164501

Colombi R, Schlüter M, and von Kameke A (2021) Three dimensional flows beneath a thin layer of 2d turbulence induced by faraday waves. Experiments in Fluids 62:1-13

Couder Y, Protiere S, Fort E, and Boudaoud A (2005) Dynamical phenomena: Walking and orbiting droplets. Nature 437:208

Francois N, Xia H, Punzmann H, Ramsden S, and Shats M (2014) Three-dimensional fluid motion in faraday waves: creation of vorticity and generation of two-dimensional turbulence. Physical Review X 4:021021

Francois N, Xia H, Punzmann H, and Shats M (2013) Inverse energy cascade and emergence of large coherent vortices in turbulence driven by faraday waves. Physical review letters 110:194501

Kelley DH and Ouellette NT (2011) Onset of three-dimensionality in electromagnetically driven thin-layer flows. Physics of Fluids 23:045103

Kokot G, Das S, Winkler RG, Gompper G, Aranson IS, and Snezhko A (2017) Active turbulence in a gas of self-assembled spinners. Proceedings of the National Academy of Sciences 114:12870-12875

Martell BC, Tithof J, and Kelley DH (2019) Comparing free surface and interface motion in electromagnetically driven thin-layer flows. Physical Review Fluids 4:043904

Saylor JR and Kinard AL (2005) Simulation of particle deposition beneath faraday waves in thin liquid films. Physics of Fluids 17:047106 von Kameke A, Huhn F, Fernández-García G, Munuzuri A, and Pérez-Muñuzuri V (2011) Double cascade turbulence and richardson dispersion in a horizontal fluid flow induced by faraday waves. Physical review letters 107:074502

von Kameke A, Huhn F, Munuzuri A, and Pérez-Muñuzuri V (2013) Measurement of large spiral and target waves in chemical reactiondiffusion-advection systems: Turbulent diffusion enhances pattern formation. Physical review letters 110:088302

Xia H and Francois N (2017) Two-dimensional turbulence in three-dimensional flows. Physics of Fluids 29:111107 